\title{
Validation by the HARP Method of a New Algorithm to Assess Left Ventricular Deformation on Standard Cine-MRI
}

\author{
Y Chenoune $^{1}$, E Deléchelle $^{1}$, E Petit $^{1}, \mathrm{~J} \mathrm{Garot}^{2}$, A Rahmouni $^{3}$ \\ ${ }^{1}$ Laboratoire Images, Signaux et Systèmes Intelligents (LISSI), Université Paris XII, Créteil, France \\ ${ }^{2}$ UMR 660 INSERM, Faculté de Médecine de Créteil, France \\ ${ }^{3}$ Département d'Imagerie Médicale, Hôpital Henri Mondor, Créteil, France
}

\begin{abstract}
We present an original algorithm to assess the deformations of the left ventricle on standard cardiac cine-MRI rather than tagged-MRI. Our results are validated by comparison with results derived from tagged image sequences that are analysed by the Harmonic Phase MRI (HARP MRI) method.

First, a segmentation process, based on a level set method is applied on a $2 D+t$ dataset of MR images to detect endocardial contours. Second, the successive segmented contours are matched using a rigid registration. We then apply a morphing process implemented with a level set approach to track the position of points belonging to the endocardial contours. Finally, local measurements of myocardial deformations are derived from the previously determined matched contours.
\end{abstract}

\section{Introduction}

Due to ischemic heart diseases, mechanical properties of the myocardium can be modified. Consequently, the quantification of the left ventricle deformations on cardiac images can provide precious information to characterize the cardiac function.

Tagged-MRI provides a grid that is superimposed on the image. The tracking of these tags is widely used to obtain cardiac deformation measurements [1, 2]. Recently, the Harmonic Phase MRI (HARP MRI) [3], was developed to derive the strain fields without tag detection and tracking and has been validated from a clinical point of view [4]. Other approaches relied with the Phase Contrast MRI [5], or extract the parietal deformations by directly analyzing untagged cardiac MRI sequences [6]. We are interested by this last approach and our original contribution consists in assessing left ventricular deformations from standard cine-MRI rather than tagged-MRI.

First, the segmentation of the endocardial borders is achieved by using a level set approach. Then, we track the successive detected contours over the time by applying a global alignment followed by a morphing procedure based on a level set approach [7]. Finally, the myocardial deformations are computed and compared to measurements obtained on the same patients by the HARP reference method from the corresponding slice levels on tagged-MR images.

\section{The standard Level Set method}

The basic concept of the level set method is to represent a $n$ dimension interface by the zero level set of a $n+1$ dimension function $\phi$ called a level set function.

Therefore, the interface is defined by the points $x(t)$ that verify the following equation :

$\phi(x(t), t)=0$

The evolution equation of $\phi$ is obtained by deriving this equation over time. By using a Hamilton-Jacobi formulation, the partial differential equation that governs the temporal evolution of the function $\phi$ can be written as follows:

$\frac{\partial \phi(x, t)}{\partial t}=g(I) \cdot\left(v_{0}+\varepsilon \kappa\right)|\nabla \phi|+\nabla g . \nabla \phi$

The first term of the equation 2 corresponds to the velocity model proposed by Caselles et al [8], where $v_{0}$ is a constant velocity imposing an expansion or a contraction depending on its sign, $\kappa$ the curvature, acting as a regularization term, $\varepsilon$ a weighting parameter and $g(I)$ a contour indicator function, defined as follows:

$g(I)=e^{-b^{*} I}$

with $b$ the decreasing constant of the exponential term. The second term of the equation 2 represents a local advection force that attracts the interface either when it comes close to the contours or when it has overstepped them. The numerical resolution of this evolution equation is based on a finite difference scheme proposed by Osher and Sethian [9]. 


\section{Segmentation and contours matching}

\subsection{Segmentation algorithm}

We directly apply the level set-based segmentation procedure on a $2 D+t$ cine-MRI dataset, in order to detect endocardial contours. The initial zero level set is a simple polygon manually drawn inside the left ventricular cavity on a still frame of the sequence. The initial level set function is iteratively updated according to the evolution equation on a narrow band [10] around the moving interface. We added the following region-based constraint to obtain contours that accurately fit the endocardial borders: $\left(I-C_{e x t}\right)^{2}-\left(I-C_{\text {int }}\right)^{2}$. Such constraints were recently added in a level set method by Chan and Vese [11]. $C_{\text {ext }}$ and $C_{\text {int }}$ are respectively the average grey level in the external region and in the internal one. The evolution equation becomes:

$$
\begin{aligned}
& \frac{\partial \phi(x, t)}{\partial t}= \\
& g(I)\left(v_{0}+\varepsilon \kappa\right)|\nabla \phi|+\nabla g . \nabla \phi+\beta\left[\left(I-C_{e x t}\right)^{2}-\left(I-C_{\text {int }}\right)^{2}\right]
\end{aligned}
$$

where $\beta \geq 0$ is a weighting parameter experimentally fixed to 10 .

\subsection{Geometric matching of the contours}

To assess cardiac deformation, a contour matching operation is required after the segmentation stage in order to track the position of points belonging to the endocardial contours over time. This matching operation combines a procedure of global alignment and a morphing of the successive detected contours.

The alignment procedure [12] realizes a rigid registration between two curves $C_{1}$ and $C_{2}$ - two successive endocardial contours- by an affine geometrical transformation controlled by a dissimilarity criterion based on 3 parameters: the Euclidean distance between two points $d_{j, k}$, the difference of local curvature of these two points $\kappa_{j, k}$ and the difference of orientation of the normal to the curves at these two points $n_{j, k}$. The measurement of dissimilarity between two points $p_{1}$ and $p_{2}$ belonging to two successive contours $C_{1}$ and $C_{2}$ is given by the following expression:

$D_{j, k}=d_{j, k} \cdot\left(\kappa_{j, k}\right)^{\gamma_{\kappa}} \cdot\left(n_{j, k}\right)^{\gamma_{n}}$

where $D_{j, k}>1$ and $\gamma_{\kappa}$ and $\gamma_{n}$ are positive weighting factors, experimentally fixed to 4 .

Taking the curve $C_{1}$ as a reference, one has to find for each point $p_{2}$ of $C_{2}$, a single point $p_{1}$ of $C_{1}$ that minimizes the dissimilarity measurement. The next processing step consists of tracking the points of a contour towards the following one, using a morphing process that we have implemented with a level set method. Given two successive contours, $C_{S}$ and $C_{D}$, we want $C_{S}$ (the source curve) to evolve towards $C_{D}$ (the destination curve). The equation of evolution is given by:

$\varphi_{t}=\left(\varphi_{D}-\varphi\right)|\nabla \varphi|$

\section{Cardiac deformation measurement and validation}

\subsection{Local deformation parameter}

In order to validate our results, we compare them with the reference HARP method results. We chose to determine the Relative Circumferential Shortening strain, denoted $R C S$. The end-diastolic contour being taken as a reference, the $R C S$ is assessed by the relative variation of length of a finite myocardial segment. For the $k^{\text {th }}$ contour of the sequence, $R C S_{k, r e f}(i, j)$ reads:

$R C S_{k, r e f}(i, j)=\frac{d_{k}(j)-d_{r e f}(i)}{d_{r e f}(i)}$

with $d_{r e f}$ the length of the end-diastolic reference contour $C_{r e f}$. For the quantitative analysis, we divided the left ventricle into 5 equiangular sectors, with a reference landmark set at the junction of the inferior septum and the right ventricle. Fig.1 shows an example of the 5 myocardial sectors. For each sector, we computed the average $R C S$ denoted $R C S_{\text {mean }}$ :

$R C S_{\text {mean }}=\frac{\sum_{\text {sector }} R C S_{k, \text { ref }}}{N}$

with $N$ the number of measurements performed for a given sector, on the endocardial segment.

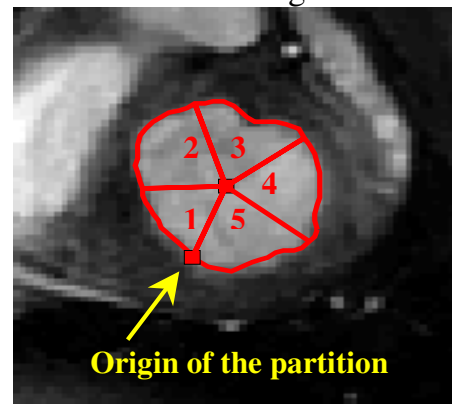

Figure 1. Division on a short axis MR image of the LV myocardium into 5 sectors, corresponding clockwise to: 1-septal, 2-anteroseptal, 3-anterior, 4-lateral and 5-inferior.

The assessment of myocardial $R C S_{\text {mean }}$ was compared to the results given by an expert using the HARP semiautomated reference method. 


\subsection{Results and validation}

All the images were provided by the Department of Medical Imaging of the Henri Mondor Hospital, Créteil, France. The assessment of $R C S_{\text {mean }}$ was performed on 7 healthy volunteers and 11 patients, studied at an average of 3 days after acute myocardial infarction (mean age $59 \pm 8$ years). There were 5 patients with anterior infarction, 6 with lateral or inferior infarcts. All patients were treated by successful primary angioplasty of the infarct-related artery that is a widely validated technique for the treatment of acute myocardial infarction. For 2 healthy volunteers, we processed a set of slices to carry out a $3 \mathrm{D}$ investigation.

Clinical validation: we propose to assess the correlation between the measurements obtained from cine-MRI by our method and those extracted from a standard visual semi-quantitative analysis of myocardial thickening performed by a blinded physician (expert cardiologist well-trained in the visual assessment of segmental cardiac contraction on cine-MRI) that graded cardiac contraction in 4 groups: normal, hypokinetic, akynetic and dyskinetic. We derived 150 measurements that are separated in 4 groups according to the classification of the physician. Figure 2 displays the 4 probability densities that represent the distribution of the maximal values of $R C S_{\text {mean }}$. We see that the automatic measurements derived from our method are well gathered in the four predefined classes. An accurate analysis of these results shows that the secondary mode appearing in the distribution of normal sectors corresponds to nonischemic sectors found in pathological cases, which was expected from current knowledge. Indeed, remote nonischemic segments are known to contract less than corresponding normal segments in controls. This preliminary clinical validation was then completed by a quantitative validation of $R C S_{\text {mean }}$.

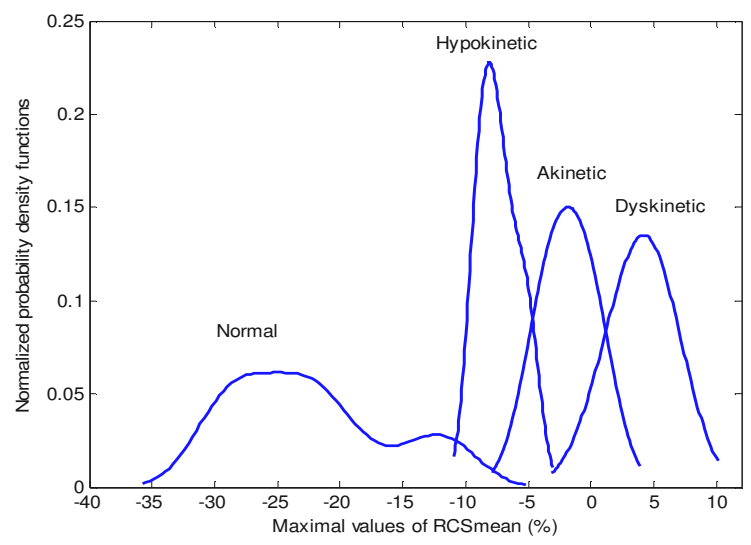

Figure 2. The probability density functions representing the distribution of the maximal values of $R C S_{\text {mean }}$ for the 4 clinical grades of segmental LV contraction as assessed visually by an expert physician blinded to the quantitative results.

Quantitative validation: this operation was realized by comparing our quantitative measurements of $R C S_{\text {mean }}$ to those derived from the HARP method. We give an example of the temporal evolution of $R C S_{\text {mean }}$ in the five myocardial sectors of a medio-ventricular short axis slice, for a healthy volunteer (fig. 3-a) and in a patient with lateral myocardial infarction (fig. 3-b). As for all the compared results, the measurements provided by the two methods are in good agreement. In the example (3-a), a clear default of contraction is showed up during the whole cardiac cycle in the lateral wall (see the flat profile of $R C S_{\text {mean }}$ for the $3^{\text {th }}$ and $4^{\text {th }}$ sectors on fig. 3-b). No lack of contraction was seen in healthy volunteers who had more homogeneous segmental myocardial contraction.

In conclusion, the quantitative comparison between our method and HARP showed a good correspondence for the whole dataset. Furthermore, our measurements of $R C S_{\text {mean }}$ were performed during the entire cardiac cycle, while those provided by HARP encompassed only the systolic phase because of the disappearance of the tag lines over time.

\section{Conclusion}

We proposed an efficient algorithm to derive accurate quantitative measurements of myocardial deformations, from standard untagged cine-MRI. One of the main advantages of our approach lies in the fact that there's no need for long acquisition of tagged-MRI. It is to our knowledge one of the first attempt to assess myocardial strain from standard cardiac cine-MRI. Our algorithm is able to detect the presence of a lack of contraction in all infarct patients and this lack of contraction corresponded well to the location of the myocardial infarction. We can discriminate dysfunctional and healthy sectors when analyzing the obtained curves.

Except for the initialization of the segmentation, which can be reduced to placing one point in the left ventricular cavity on one image of the $2 D+t$ set, the entire procedure is fully automated. The computational time after the segmentation and the geometric matching of a $2 D+t$ set of ten images was of the order of two minutes, on a standard personal computer (Xeon Processor $2.4 \mathrm{GHz}, 1$ Go RAM). We also demonstrate the robustness of this novel method of assessing cardiac deformation which is readily available for routine clinical examinations.

Complementary works are being carried out to extend this method of segmentation and matching for both endocardial and epicardial borders. 
RCSmean (\%)
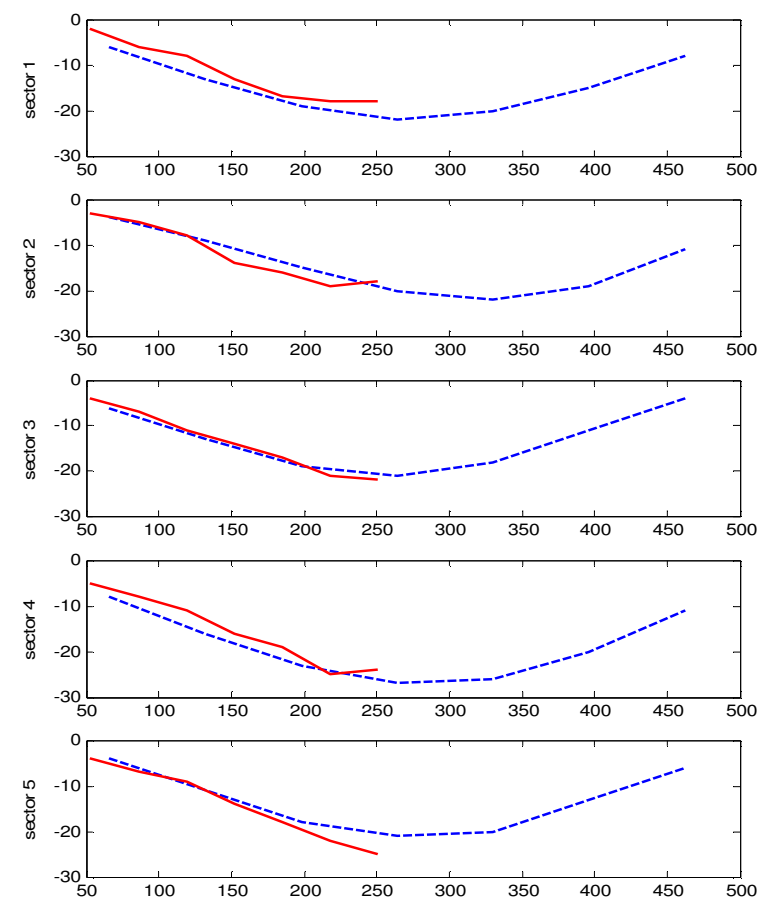

(a)
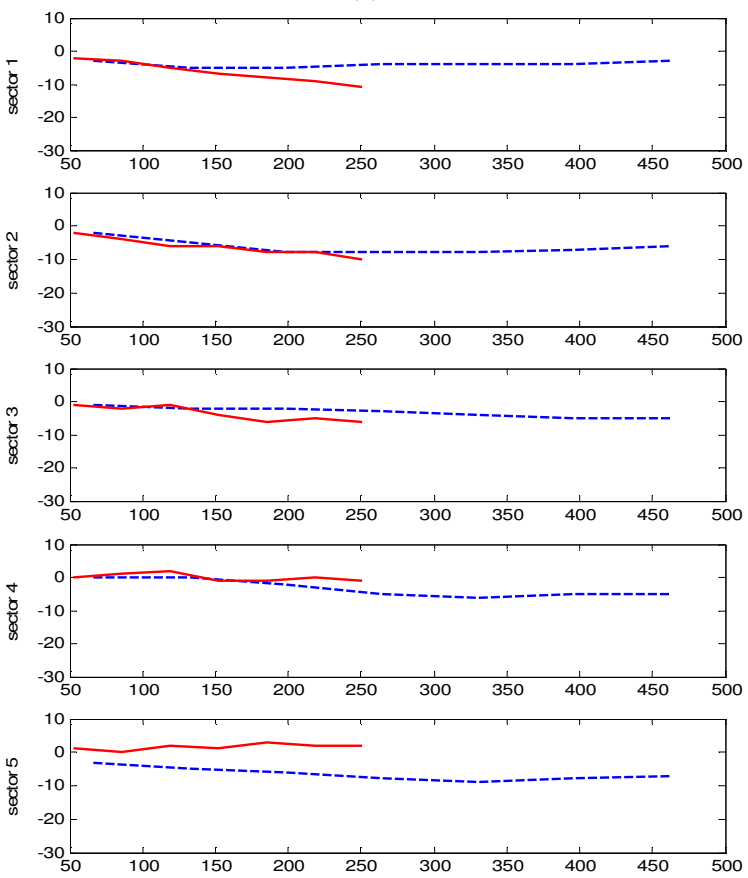

(b)

Figure 3. Temporal evolution of the mean value of $R C S$ $\left(R C S_{\text {mean }}\right.$ in percentage) in the 5 myocardial sectors in a midventricular short-axis slice using the level set approach from cine-MRI (dotted line) and the HARP technique from taggedMRI (unbroken line) in a healthy volunteer (a) and in a patient with an anterior myocardial infarction (b).

\section{References}

[1] Young A, Axel L. Three-Dimensional Motion and Deformation of the Heart Wall : Estimation with Spatial Modulation of Magnetization : a Model-Based Approach. Radiology 1992;185(1):241-247.

[2] O'Dell W, Moore C, Hunter W, Zerhouni E, Veigh EM. Three Dimensional Myocardial Deformations : Calculation with Displacement Field Fitting to Tagged MR Images. Radiology 1995;195:829-835.

[3] Osman N, McVeigh E, Prince J. Imaging Heart Motion using Harmonic Phase MRI. IEEE Trans Med Imaging 2000;19(3):186-202.

[4] Garot J, Bluemke D, Osman N, Rochitte C, McVeigh E, Zerhouni E, et al. Fast Determination of Regional Myocardial Strain Fields from Tagged Cardiac MR Images using Harmonic Phase (HARP) MRI. Circulation 2000;101:981-988.

[5] Pelc NJ, Herfkens R, Pelc L. 3D analysis of myocardial motion and deformation with phase contrast cine MRI. In: Proceedings of the $11^{\text {th }}$ Annual SMRM; 1992; Berlin; 1992.

[6] Paragios N. A Variational Approach for the Segmentation of the Left Ventricle in MR Cardiac Images. International Journal of Computer Vision 2002;50(3):345-362.

[7] Bertalmio M, Sapiro G, Randall G. Morphing Active Contours. IEEE Trans on Pattern Analysis and Machine Intelligence 2000;22(7).

[8] Caselles V, Kimmel R, Sapiro G. Geodesic Active Contours. International Journal of Computer Vision 1997;22(1):61-79.

[9] Osher SJ, Sethian JA. Fronts Propagating with CurvatureDependent Speed : Algorithms based on Hamilton-Jacobi Formulations. Journal of Computational Physics 1988;79:12-49.

[10] Adalsteinsson D, Sethian JA. A Fast Level Set Method for Propagating Interfaces. Journal of Computational Physics 1995;118(2):269-277.

[11] Chan TF, Vese LA. Active Contours Without Edges. IEEE Transactions On Image Processing 2001;10(2)(2):266 -277.

[12] HILA-BENHAJEL K. Étude et Modélisation de la Fonction Cardiaque par Analyse Numérique d'Images. Phd thesis, LERISS, Université Paris XII Val-de-Marne, Créteil, France 2003.

Address for correspondence

\begin{tabular}{|c|c|}
\hline Names, e-mail address & Full postal adresses \\
\hline $\begin{array}{l}\text { Y. CHENOUNE, E. } \\
\text { DeLECHELlE, E. PETIT } \\
\text { chenoune, delechelle, } \\
\text { petit @ univ-paris12.fr }\end{array}$ & $\begin{array}{c}\text { LISSI } \\
\text { Université Paris XII } \\
\text { 61, avenue du Général de } \\
\text { Gaulle, 94010 Créteil France }\end{array}$ \\
\hline $\begin{array}{c}\text { J. GAROT } \\
\frac{\text { jerome.garot@ @mn.ap- }}{\underline{\text { hop-paris.fr }}}\end{array}$ & $\begin{array}{c}\text { UMR } 660 \text { INSERM, Faculté de } \\
\text { Médecine Créteil, 51, avenue } \\
\text { Maréchal de Lattre de Tassigny. } \\
94010 \text { Créteil France }\end{array}$ \\
\hline $\begin{array}{c}\begin{array}{c}\text { Alain RAHMOUNI } \\
\text { alain.rahmouni@hmn }\end{array} \\
\underline{\text { ap-hop-paris.fr }}\end{array}$ & $\begin{array}{c}\text { Département d'Imagerie } \\
\text { Médicale, Hôpital Henri Mondor, } \\
\text { 51, avenue du Maréchal de Lattre } \\
\text { de Tassigny. } 94010 \text { Créteil. France }\end{array}$ \\
\hline
\end{tabular}

